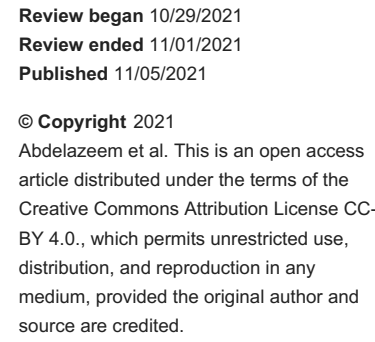

\section{Light Chain (AL) Cardiac Amyloidosis: A Diagnostic Dilemma}

\author{
Basel Abdelazeem ${ }^{1}$, Nouraldeen Manasrah ${ }^{2}$, Amman Yousaf ${ }^{3}$, Rudin Gjeka ${ }^{4}$, Arvind Kunadi ${ }^{1}$ \\ 1. Internal Medicine, McLaren Health Care, Michigan State University, Flint, USA 2. Internal Medicine, Detroit Medical \\ Center (DMC) Sinai-Grace Hospital, Detroit, USA 3. Internal Medicine, McLaren Health Care, Flint, USA 4. Cardiology, \\ McLaren Regional Medical Center, Flint, USA
}

Corresponding author: Basel Abdelazeem, basel.abdelazeem@mclaren.org

\begin{abstract}
Amyloidosis is a clinical condition characterized by amyloid fibril deposition into different organ systems. The most common types are light chain (AL) amyloidosis and transthyretin amyloidosis (ATTR) amyloidosis. Amyloidosis involves the heart with an incidence of 1.38 to 3.69 per 100,000 person-years and a prevalence of 14.85 per 100,000 person-years between 2004 and 2018. Diagnosis of cardiac amyloidosis can be made through cardiac imaging, including cardiac magnetic resonance imaging (CMR) and 99mTc-labeled pyrophosphate (PYP) cardiac scan. However, a tissue biopsy is frequently needed to confirm the diagnosis. Herein, we report such a case of cardiac amyloidosis. The patient presented with pericardial effusion and acute kidney injury as the initial presentation. The presumptive diagnosis was ATTR amyloidosis, but the endomyocardial biopsy confirmed the diagnosis of AL amyloidosis. The patient was started on bortezomib, cyclophosphamide, and dexamethasone therapy. We aimed to highlight the different diagnostic modalities of cardiac amyloidosis and the importance of obtaining tissue biopsy to confirm the amyloidosis type before starting the treatment.
\end{abstract}

Categories: Cardiac/Thoracic/Vascular Surgery, Cardiology, Internal Medicine

Keywords: case report, cardiac imagings, transthyretin, light chain, amyloidosis

\section{Introduction}

Amyloidosis refers to the deposition of extracellular protein into different body organs. Amyloidosis has 22 different types of localized forms and 18 types of systemic forms [1]. The most common systemic forms are light chain (AL) amyloidosis and transthyretin amyloidosis (ATTR) [2]. The clinical manifestations depend on organ involvement. Cardiac amyloidosis is becoming a common condition with an incidence of 1.38 to 3.69 per 100,000 person-years and a prevalence of 14.85 per 100,000 person-years between 2004 and 2018 [3]. Cardiac amyloidosis presents with symptoms and signs of heart failure, conduction system abnormalities, and ischemia. Cardiac involvement was reported in $88 \%$ of patients with AL amyloidosis compared to $26 \%$ of patients with ATTR [4]. Our case highlights the importance of early diagnosis to improve the patients' quality of life and discusses the different diagnostic modalities of amyloidosis.

\section{Case Presentation}

A 72-years-old African American female with a past medical history of hypothyroidism presented to our hospital with dyspnea, orthopnea, paroxysmal nocturnal dyspnea, and leg swelling. The patient denied chest pain, palpitation, fever, or night sweats. The patient denied any heart disease, and she was never on any medications for heart disease. On physical exam, vital signs were blood pressure 146/96 mmHg, heart rate 91 beats per minute, $98.1 \mathrm{~F}$ temperature, and the patient was saturating $94 \%$ on a two-liter supplemental oxygen using a nasal cannula. The patient had distant heart sounds but regular S1 and S2, no murmurs, rubs, or gallops. The patient had an equal breathing sound bilateral, without any wheezes or ronchi. Extremities revealed bilateral 2+ edema pedal edema.

Laboratory workup is summarized in Table 1. Electrocardiogram (EKG) revealed prolonged PR interval and type one atrioventricular block (Figure 1A). Computerized tomography of the chest revealed moderate cardiomegaly, pericardial effusion, and slight right pleural effusion (Figure 1B). An echocardiogram revealed a left ventricular (LV) ejection fraction of $25 \%-30 \%$, severely increased left atrial volume $48.6 \mathrm{ml} / \mathrm{m} 2$, moderate pulmonary hypertension with right ventricular systolic pressure of $53.66 \mathrm{mmHg}$, and a moderate to large pericardial effusion behind LV without tamponade (Figure 1C) (Video 1). Cardiothoracic surgery was consulted, and the patient underwent a pericardial window with about $600 \mathrm{ml}$ of fluid removed, the fluid was sent for Congo red stain, and it was negative. The cardiothoracic team placed a chest tube in the pericardial cavity which was removed later when the patient was more stable. 


\section{Cureus}

\begin{tabular}{|c|c|c|}
\hline Lab & Value & References \\
\hline WBC Count & $3.66 \mathrm{~L}$ & $4.50-11.00 \times 10 * 3 / u L$ \\
\hline Hemoglobin & $10.9 \mathrm{~L}$ & $12.0-15.7 \mathrm{~g} / \mathrm{dL}$ \\
\hline Platelet Count & 179 & $140-440 \times 10^{*} 3 /$ uL \\
\hline BUN & 19 & $7-22 \mathrm{mg} / \mathrm{dL}$ \\
\hline Creatinine & $2.11 \mathrm{H}$ & $0.50-1.50 \mathrm{mg} / \mathrm{dL}$ \\
\hline C Reactive Protein & $4.3 \mathrm{H}$ & $0.0-0.8 \mathrm{mg} / \mathrm{dL}$ \\
\hline BNPEP Brain Natriuretic Peptide & $>5000 \mathrm{H}$ & $2-100 \mathrm{pg} / \mathrm{mL}$ \\
\hline Troponin-I High Sensitivity & $0.1880 \mathrm{H}$ & $0.0000-0.0400 \mathrm{ng} / \mathrm{mL}$ \\
\hline Kappa FLC-Serum & $2.96 \mathrm{H}$ & $0.33-1.94 \mathrm{mg} / \mathrm{dL}$ \\
\hline Lambda FLC-Serum & $9.68 \mathrm{H}$ & $0.57-2.63 \mathrm{mg} / \mathrm{dL}$ \\
\hline Kap/Lam FLC Ratio-Ser & 0.306 & $0.260-1.650$ \\
\hline Serum Immunofixation & IgG lambda paraprotein & NA \\
\hline Immunoglobulin IgA & 175.0 & $60.0-350.0 \mathrm{mg} / \mathrm{dL}$ \\
\hline Immunoglobulin IgG & $1950.0 \mathrm{H}$ & $700.0-1600.0 \mathrm{mg} / \mathrm{dL}$ \\
\hline Immunoglobulin IgM & 46.2 & $40.0-280.0 \mathrm{mg} / \mathrm{dL}$ \\
\hline Antineutrophil Cytoplasmic Antibodies- MPO unit & $<0.2$ & Negative < 1.0 Al; Positive $\geq 1.0 \mathrm{Al}$ \\
\hline Antineutrophil Cytoplasmic Antibodies- PR3 unit & $<0.2$ & Negative $<1.0 \mathrm{Al}$; Positive $\geq 1.0 \mathrm{Al}$ \\
\hline Vitamin B12 & 764.0 & 200.0-944.0 pg/mL \\
\hline Erythropoietin & 7.36 & 2.00-30.00 mlU/mL \\
\hline Ferritin & $372.7 \mathrm{H}$ & $10.0-291.0 \mathrm{ng} / \mathrm{mL}$ \\
\hline Total Iron Binding Capacity & 270 & $228-460$ ug/dL \\
\hline
\end{tabular}

\section{TABLE 1: Laboratory workup}

FLC: free light chain; WBC: white blood cell; BUN: blood urea nitrogen; L: low; H: high; NA: non-applicable. 


\section{Cureus}
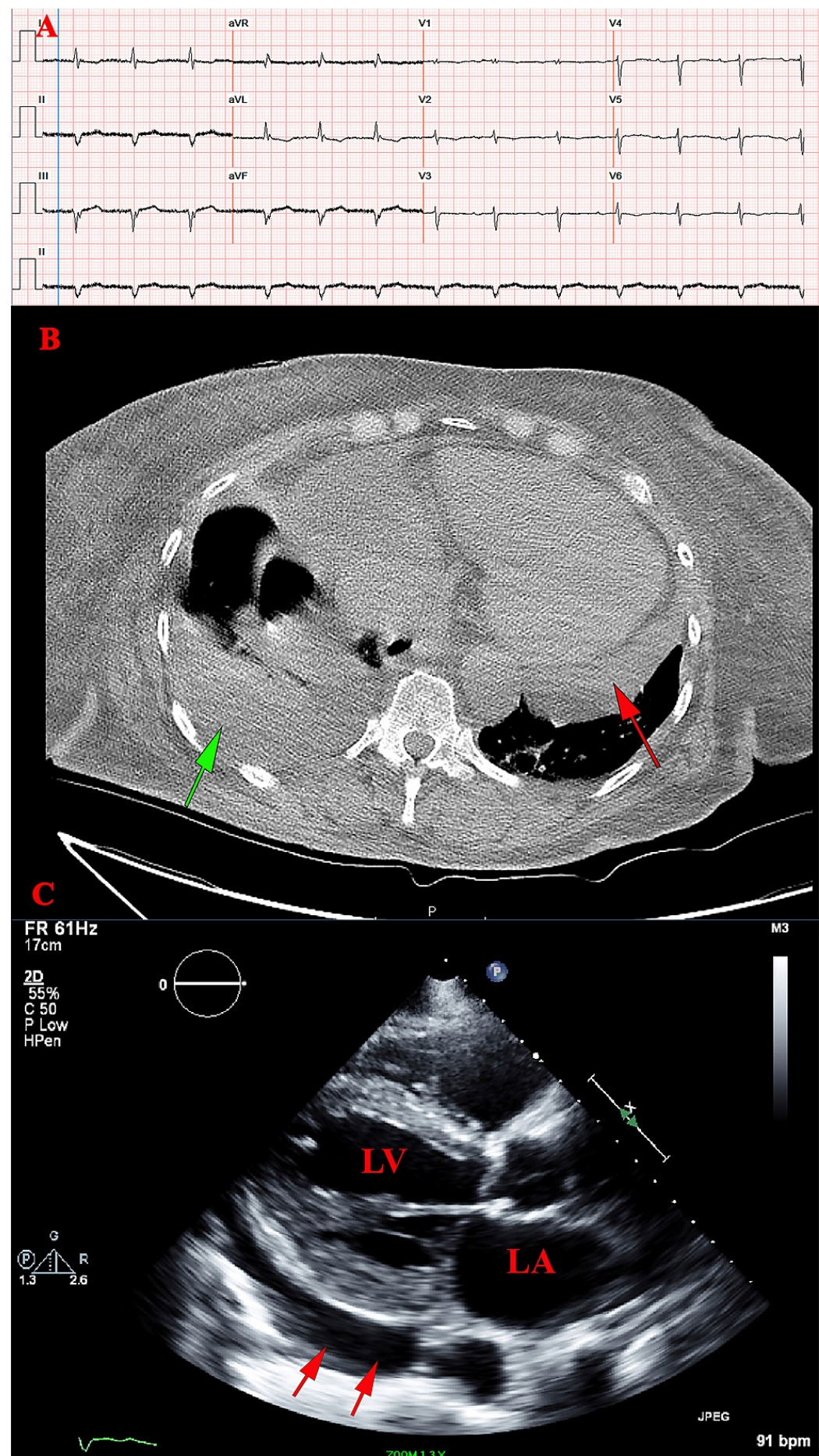

\section{FIGURE 1: Patient's work up}

A) EKG revealed prolonged PR interval and type one atrioventricular block. B) Computerized tomography of the chest revealed moderate cardiomegaly, pericardial effusion, and small right pleural effusion; red arrow is pointing to pericardial effusion; the green arrow is pointing to pleural effusion. C) Transthoracic 2D echocardiogram revealed a moderate to large pericardial effusion behind LV without tamponade; arrows are pointing to the pericardial effusion.

LV: left ventricle; LA: left artium. 


\section{Cureus}

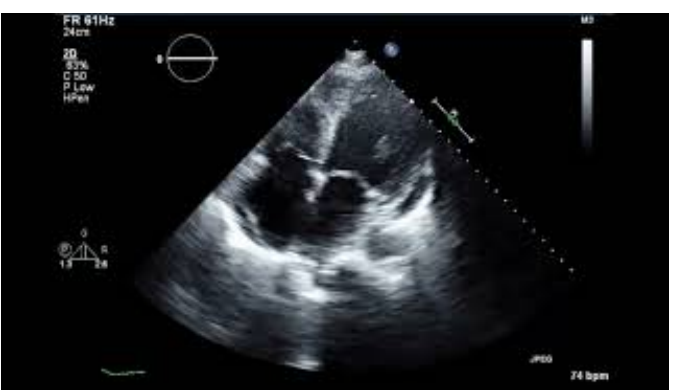

\section{VIDEO 1: Transthoracic echocardiogram}

Transthoracic 2D echocardiogram revealed a left ventricular ejection fraction of $25 \%-30 \%$ and severely increased left atrial volume $48.6 \mathrm{ml} / \mathrm{m} 2$

View video here: https://youtu.be/FoVp2qsnfJ8

On telemetry, multiple pauses were noted, with the most prolonged pause of $2.5 \mathrm{sec}$ and premature ventricular contractions. The patient was not able to undergo cardiac catheterization due to acute kidney injury. The patient was discharged with a plan of two-week event monitor, life vest, outpatient cardiac magnetic resonance imaging (CMR), and 99mTc-labeled pyrophosphate (PYP) cardiac scan.

CMR showed the left and right ventricles are moderately dilated. Mildly increased LV thickness at the basal septal wall $13 \mathrm{~mm}$ with prominent trabeculations in the mid and apical segments. The LV ejection fraction is $33 \%$. High T1 value (average 1240), which may indicate infiltrative myocardial disease (Figure $2 A$ ). The patient underwent CT guided percutaneous core biopsy of the abdominal fat pad, and it was negative for both amyloid deposit and Congo red staining. The PYP scan was strongly suggestive of ATTR amyloidosis with grade 3 of the visual interpretation (Figure $2 B$ ). A genetic test was sent and was negative. Endomyocardial biopsy was done and revealed AL amyloidosis (Figure 3). The patient was started on bortezomib, cyclophosphamide, and dexamethasone therapy. 


\section{Cureus}



\section{FIGURE 2: Caridiac imagings}

A) Cardiac magnetic resonance imaging (CMR) showed mildly increased LV thickness at the basal septal wall 13 $\mathrm{mm}$ with prominent trabeculations in the mid and apical segments (the green arrows). High T1 value (average 1240), which may indicate infiltrative myocardial disease. B) 99mTc-labeled pyrophosphate (PYP) cardiac scan showed features strongly suggestive of transthyretin cardiac amyloidosis with grade three of the visual interpretation (the green circle indicates the degree cardiac tissue, the blue circle indicates the degree of rib tissue) 


\section{Cureus}

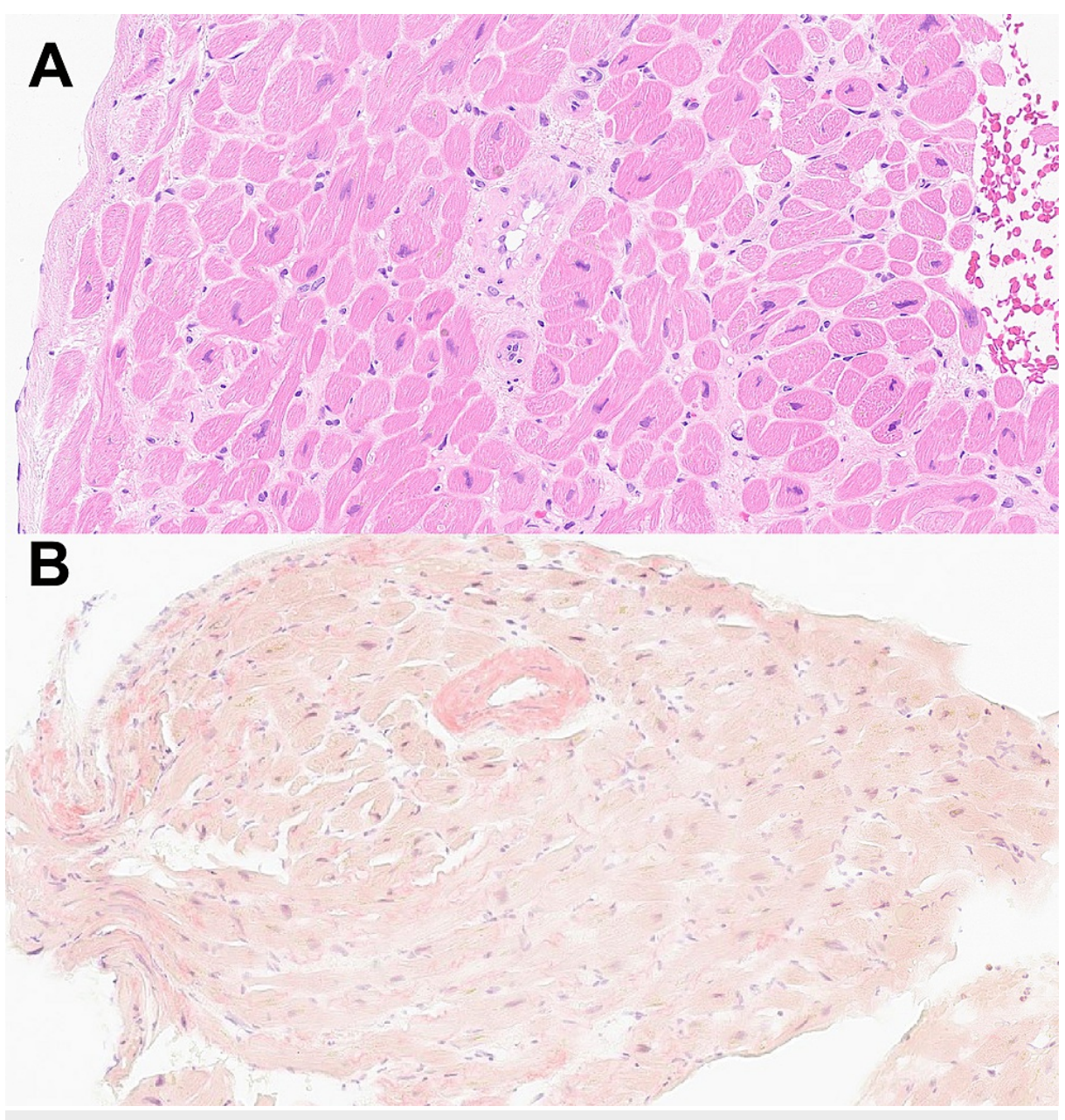

FIGURE 3: Histopathology of the endomyocardial biopsy

Histopathology with 10x power showed amyloid deposits between the cardiac muscles and around the blood vessels. A) hematoxylin and eosin stain. B) Congo red stain.

\section{Discussion}

Cardiac amyloidosis presents with symptoms and signs of restrictive cardiomyopathy and right-sided heart failures, brady or tachyarrhythmia, and angina [5,6]. Extracardiac manifestations include fatigue, weight loss, peripheral neuropathy, carpal tunnel syndrome, nephrotic syndrome, macroglossia, and periorbital purpura [7].

Laboratory test abnormalities include elevated brain natriuretic peptide, troponin, and proteinuria. Monoclonal proteins testing is essential for diagnosis, and the following test should be performed serum kappa/lambda free light chain ratio, serum protein immunofixation, and urine protein immunofixation. EKG criteria include pseudo-infarction patterns, low QRS voltage, and conduction system abnormalities [8].

The echocardiogram is the initial diagnostic test of choice, and the earliest finding is relative apical sparing of longitudinal strain. Other findings include ventricular hypertrophy, thickening of the inter-atrial septum and the valves, dilated atrium, and speckled myocardium [9]. CMR is the test of choice to diagnose cardiac amyloidosis with a characteristic appearance on the late gadolinium enhancement over the entire subendocardial circumference. PYP cardiac scan has a sensitivity of $82 \%$ and specificity of $98.8 \%$ for cardiac amyloidosis if scintigraphy revealed grade 1, 2, or 3, also it helps to distinguish ATTR from AL amyloidosis [10]. The presence of grade 2 or 3 scintigraphy in patients without monoclonal protein is $100 \%$ specific for ATTR amyloidosis [11].

Our patient had the symptoms and signs of heart failure, elevated monoclonal protein, EKG finding consistent with cardiac amyloidosis, and pericardial effusion on imaging which make cardiac amyloidosis one of the differential diagnoses. However, the CMR and PYP scans were not conclusive about the diagnosis, which warranted a tissue biopsy. Although our patient had a PYP scan that was suggestive of ATTR cardiac amyloidosis, the endomyocardial biopsy revealed AL amyloidosis. 
Cardiac amyloidosis has a poor prognosis, but chemotherapy, stem cell transplantation, and cardiac transplantation can improve the survival rate in a selected patient. Li et al. compared placebo versus tafamidis in ATTR cardiomyopathy patients and reported that tafamidis reduced all-cause mortality by $30 \%$ compared to placebo [12]. In contrast, The main regime for AL amyloidosis is bortezomib, cyclophosphamide, and dexamethasone. Kastritis et al. evaluated the addition of daratumumab to bortezomib, cyclophosphamide, and dexamethasone to treat AL amyloidosis. They reported increased survival and higher complete hematological response. Thus daratumumab can be considered in resistant cases; however, more studies are needed to confirm this finding [13]. Thus, a definitive diagnosis should be made before starting the treatment plan, and the management varies depending on the type of amyloidosis.

\section{Conclusions}

Cardiac amyloidosis may be caused by AL or ATTR amyloidosis. The diagnosis of cardiac amyloidosis can be challenging through cardiac imaging, and tissue biopsy may be required to confirm the diagnosis. We present a case of cardiac amyloidosis which was presumptively diagnosed with ATTR amyloidosis depending on the PYP scan, but the endomyocardial biopsy revealed AL amyloidosis. The clinicians should consider different types of amyloidosis and reach a definitive diagnosis before starting the treatment plans.

\section{Additional Information \\ Disclosures}

Human subjects: Consent was obtained or waived by all participants in this study. N/A issued approval N/A. Conflicts of interest: In compliance with the ICMJE uniform disclosure form, all authors declare the following: Payment/services info: All authors have declared that no financial support was received from any organization for the submitted work. Financial relationships: All authors have declared that they have no financial relationships at present or within the previous three years with any organizations that might have an interest in the submitted work. Other relationships: All authors have declared that there are no other relationships or activities that could appear to have influenced the submitted work.

\section{References}

1. Benson MD, Buxbaum JN, Eisenberg DS, et al.: Amyloid nomenclature 2020: update and recommendations by the International Society of Amyloidosis (ISA) nomenclature committee. Amyloid. 2020, 27:217-22. 10.1080/13506129.2020.1835263

2. Ravichandran S, Lachmann HJ, Wechalekar AD: Epidemiologic and survival trends in amyloidosis, 19872019. N Engl J Med. 2020, 382:1567-8. 10.1056/NEJMc1917321

3. Sepehrvand N, Youngson E, Fine N, et al.: The incidence and prevalence of cardiac amyloidosis in a large community-based cohort in Alberta, Canada. J Card Fail. 2021, 10.1016/j.cardfail.2021.08.016

4. Gertz MA, Benson MD, Dyck PJ, et al.: Diagnosis, prognosis, and therapy of transthyretin amyloidosis . J Am Coll Cardiol. 2015, 66:2451-66. 10.1016/j.jacc.2015.09.075

5. Abdelazeem B, Malik B, Baral N, Gjeka R, Kunadi A: A case report of sick sinus syndrome as an initial presentation of primary amyloidosis. Cureus. 2021, 13:e13922. 10.7759/cureus.13922

6. Garg P, Gupta R, Hsi DH, Sheils LA, DiSalle MR, Woodlock TJ: Hypertrophic cardiomyopathy and symptomatic conduction system disease in cardiac amyloidosis. South Med J. 2006, 99:1390-2. 10.1097/01.smj.0000251327.38080.f1

7. Fine NM, Davis MK, Anderson K, et al.: Canadian Cardiovascular Society/Canadian Heart Failure Society joint position statement on the evaluation and management of patients with cardiac amyloidosis. Can J Cardiol. 2020, 36:322-34. 10.1016/j.cjca.2019.12.034

8. González-López E, Gagliardi C, Dominguez F, et al.: Clinical characteristics of wild-type transthyretin cardiac amyloidosis: disproving myths. Eur Heart J. 2017, 38:1895-904. 10.1093/eurheartj/ehx043

9. Maurer MS, Elliott P, Comenzo R, Semigran M, Rapezzi C: Addressing common questions encountered in the diagnosis and management of cardiac amyloidosis. Circulation. 2017, 135:1357-77. 10.1161/CIRCULATIONAHA.116.024438

10. Brownrigg J, Lorenzini M, Lumley M, Elliott P: Diagnostic performance of imaging investigations in detecting and differentiating cardiac amyloidosis: a systematic review and meta-analysis. ESC Heart Fail. 2019, 6:1041-51. 10.1002/ehf2.12511

11. Gillmore JD, Maurer MS, Falk RH, et al.: Nonbiopsy diagnosis of cardiac transthyretin amyloidosis . Circulation. 2016, 133:2404-12. 10.1161/CIRCULATIONAHA.116.021612

12. Li B, Alvir J, Stewart M: Extrapolation of survival benefits in patients with transthyretin amyloid cardiomyopathy receiving tafamidis: analysis of the tafamidis in Transthyretin Cardiomyopathy Clinical Trial. Cardiol Ther. 2020, 9:535-40. 10.1007/s40119-020-00179-2

13. Kastritis E, Palladini G, Minnema MC, et al.: Daratumumab-based treatment for immunoglobulin light-chain amyloidosis. N Engl J Med. 2021, 385:46-58. 10.1056/NEJMoa2028631 